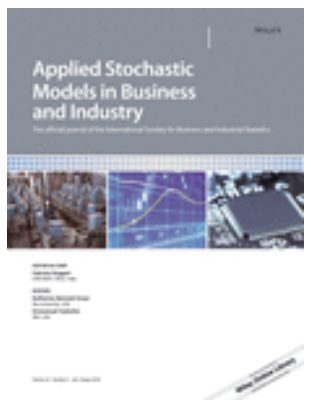

\title{
A distribution-based method to gauge market liquidity through scale invariance between investment horizons
}

\begin{tabular}{|c|c|}
\hline Journal: & Applied Stochastic Models in Business and Industry \\
\hline Manuscript ID & ASMB-19-102.R1 \\
\hline Wiley - Manuscript type: & Research Article \\
\hline $\begin{array}{r}\text { Date Submitted by the } \\
\text { Author: }\end{array}$ & 04-Nov-2019 \\
\hline Complete List of Authors: & $\begin{array}{l}\text { Bianchi, Sergio; University of Cassino and Southern Lazio, Economics } \\
\text { and Law } \\
\text { Pianese, Augusto; University of Cassino and Southern Lazio, Economics } \\
\text { and Law } \\
\text { Frezza, Massimiliano; University of Cassino and Southern Lazio, } \\
\text { Economics and Law }\end{array}$ \\
\hline Keywords: & $\begin{array}{l}\text { Self-similarity, Scale invariance, Fractal Market Hypothesis, Liquidity, } \\
\text { Investment horizons }\end{array}$ \\
\hline
\end{tabular}

\section{SCHOLARONE \\ Manuscripts}




\title{
A distribution-based method to gauge market liquidity through scale invariance between investment horizons
}

\author{
Sergio Bianchi ${ }^{1 * \circ}$, Augusto Pianese* and Massimiliano Frezza* \\ * QuantLab, Dept. of Economics and Law, University of Cassino (Italy) \\ ${ }^{\circ}$ Int'l Affiliate Professor, Dept. Finance and Risk Engineering, \\ Tandon School of Engineering, New York University (USA)
}

\begin{abstract}
A nonparametric method is developed to detect self-similarity among the rescaled distributions of the log-price variations over a number of time scales. The procedure allows to test the statistical significance of the scaling exponent that possibly characterizes each pair of time scales, and to analyze the link between self-similarity and liquidity, the core assumption of the Fractal Market Hypothesis (FMH). The method can support financial operators in the selection of the investment horizons as well as regulators in the adoption of guidelines to improve the stability of markets. The analysis performed on the S\&P500 reveals a very complex, time-changing scaling structure, which confirms the link between market liquidity and self-similarity.
\end{abstract}

Keywords: Self-similarity, Scale invariance, Fractal Market Hypothesis, Liquidity, Investment horizons

\section{Introduction}

The idea that some scale invariance principle can rule many natural as well as artificial complex phenomena such as the branching of a tree, the cosmic microwave background, or the financial markets and other self-organized collective systems, is both fascinating and challenging and originated an impressive number of contributions in many fields in the last century. Scale invariance, also referred to as self-similarity, is the property that depicts those phenomena which preserve the same (geometric or probabilistic) structure regardless the degree of magnification on a spacial or time dimension. This is a peculiar feature of fractals, the expedient that nature uses to ensure the stability of complex systems. Self-similarity characterizes many stochastic processes: the Brownian motion (Bm) or the Bessel process, self similar with exponent $\frac{1}{2}$, the fractional Brownian motion (fBm), self similar with exponent $H \in[0,1)$, the $\alpha$-stable Lévy motion (sLm), self-similar with exponent $\frac{1}{\alpha}, \alpha \in(0,1)$, or - broadly speaking - the class of generalized Hermite processes, which along with some of the processes previously mentioned, includes as special cases the Rosenblatt process and other non Gaussian stochastic processes.

\footnotetext{
${ }^{1}$ Corresponding author: sergio.bianchi@unicas.it
} 
In finance, the idea that scale invariance constitutes a pattern of the price dynamics is not new and - as for natural systems - has much to do with the notion of equilibrium, whatever is the meaning given to this multifaceted word. When the notion of equilibrium is intended in the sense of an informationally efficient market excluding arbitrage opportunities, the celebrated Efficient Market Hypothesis (EMH) [16] [36] states that the discounted price is a martingale, and the link with self-similarity is established by the paradigmatic model Brownian motion, which is indeed a continuous time, $\frac{1}{2}$-self similar martingale. Precisely this property legitimates some rules of thumb frequently and widely employed by practitioners, such as the well-known law of " $t$ power $\frac{1}{2}$ ", used to annualize volatility or to scale the Value at Risk on larger time horizons. Other examples of indirect application of the scaling laws in financial markets are the variance ratios, among the most widely used indexes that constitute also the basis of the well-known Market Efficiency Coefficient (MEC), that is the ratio of long-term variance of logarithmic returns to shorter period returns variance [20].

Since the pioneering works of Mandelbrot [29] [31], a plethora of contributions documented scaling laws and self-similarity in the price fluctuations (see [30], [11], [13], or [32] for comprehensive discussions), but the role of scale invariance in financial markets has experienced a renewed interest in the recent years for practical and theoretical reasons: on the one side, the growing technology capabilities allow to analyze in depth the abundance of high-frequency data sets; on the other side, the frequency and the magnitude of the financial crises, which occur despite the increased informational efficiency, make the theory of efficient markets more and more questionable, and induce a search for more sophisticated approaches capable to describe effectively the complex dynamics of real markets.

Among the models proposed to overcome the limits of the EMH, the Fractal Market Hypothesis (FMH) introduced by [33] deserves a special attention for its realism in the description of the market mechanism. Its basic principle is that heterogeneity of market participants ultimately rests on the diversity of the time horizons they choose for their trading activities. Indeed, in normal conditions, operators act on heterogeneous investment horizons, ranging from fractions of a second, to minutes (intraday traders), to days, until months or even years (institutional investors, pension funds etc.). Using the words of [24] "[...] Investors with different investment horizons treat the inflowing information differently and their reaction is correspondingly distinct (market participants with the short investment horizon focus on the technical information and crowd behavior of other market participants, whereas investors with the long investment horizon base their decisions on the fundamental information and care little about the crowd behavior). Specific information can be a selling signal for the short-term investor but an opportunity to buy for the long-term investor, and vice versa. [...]". By virtue of this mechanism, the proper functioning of a market is assured when each relevant time horizon is populated by a share of market participants sufficient to close trades, and hence to provide liquidity. If, for some reason, market activity masses only on a small fraction of horizons (typically the shorter ones, when uncertainty predominates), the incoming information tend to be evaluated similarly by operators; as a consequence, the trading activity slows down or even freezes because the probability grows that the trading orders are not matched by orders of opposite sign. 
Several contributions have dealt with the FMH, with the aim to transpose it in a rigorous mathematical model (see, e.g., [35] and [40]) or, more often, to test its descriptive potential ([26], [15], [21]), or also to study the relation between market illiquidity and turbulent periods, mostly with regard to the 2007 - 2009 global financial crisis ([24], [25], [14]). Generally, the findings attained using different methodologies (DFA, Wavelet power spectra, variance scaling, R/S analysis) confirm the capability of FMH to describe the market behavior more effectively than the mainstream EMH.

Nonetheless, to the authors' knowledge, the methods used in literature to test scale invariance mostly consider single moments, along with their possible transforms, rather than the whole distributions of price variations. This can be misleading, because self-similarity is defined in terms of distributions, not in terms of their individual moments. Hence scale invariance could also not hold even when it holds for some specific moments; as an example, if the variance scales but the mean does not, judging only by the second-order moment could lead to neglect potential stochastic dominance effects. Furthermore, none of the methods aiming to validate or confute the FMH has studied so far the contribution of individual investment horizons to the overall liquidity of the market. This is precisely the main motivation and the main contribution of this paper, in which the scaling properties and their consequences on the financial market are studied by means of a methodology which compares the whole distributions of the log-returns related to all the pairs of investment horizons. Our empirical analysis on the stock index S\&P500 suggests that the dichotomy between scaleinvariance/liquidity and scale-dependence/illiquidity is indeed too restrictive, since a much more complex dynamics emerges from data exploration. The approach we suggest can direct and support not only the decisions of market operators in the selection of the time horizons with higher liquidity, but also the regulators' policies aiming at stabilizing the markets during crisis periods, for example by liquidity provision.

The remainder of this paper is organized as follows: Section 2 recalls the main definitions and theorems that will be implemented in the methodology, described in Section 4. Section 3 illustrates the market mechanism that links distribution, self-similarity and liquidity in the light of the FMH. Section 5 is devoted to the analysis of the stock index S\&P500. Section 6 concludes.

\section{Distribution scaling and Self-similarity}

\subsection{Theoretical background}

To introduce the link between market liquidity and self-similarity, the following basic definitions and theorems are needed.

Definition 2.1. The $\mathbb{R}^{d}$-valued stochastic process $\{X(t), t \geq 0\}$, nontrivial and stochastically continuous at $t=0^{2}$, is said to be self-similar with exponent $H_{0}$ ( $H_{0}$-ss in short) if, denoted

\footnotetext{
${ }^{2}$ Recall that $\{X(t), t \geq 0\}$ is said to be

- stochastically continuous at $t$, if for any $\epsilon>0, \lim _{h \rightarrow 0} P\{|X(t+h)-X(t)|>\epsilon\}=0$;
} 
by $\stackrel{d}{=}$ the equality between the finite-dimensional distributions, for any $a>0$, there exists a unique exponent $H_{0} \geq 0$ such that

$$
\{X(a t)\} \stackrel{d}{=}\left\{a^{H_{0}} X(t)\right\} .
$$

Remark 2.1. If $\{X(t), t \geq 0\}$ is $H_{0}$-ss and $H_{0}>0$, then $X(0)=0$ almost surely.

Definition 2.2. The second order stochastic process $\{X(t), t \geq 0\}$ is wide sense $H_{0}$-ss if for any $a>0$ :

a) $\mathbb{E}[X(a t)]=a^{H_{0}} \mathbb{E}[X(t)], \quad t>0$

b) $\mathbb{E}[X(a t) X(a s)]=a^{2 H_{0}} \mathbb{E}[X(t) X(s)], \quad t, s>0$.

Definition 2.2 weakens Definition 2.1 along two directions: instead of the whole distribution it considers only the first two moments, and it is limited to processes with finite second order moments (e.g., it excludes non-Gaussian stable processes). However, self-similarity is often tested through Definition 2.2, due to its ease of implementation.

If equality (1) holds just for some $a \in(0,1) \cup(1, \infty)$, then $\{X(t), t \geq 0\}$ is said to be semi-selfsimilar. Finally, if

$$
\{X(a t)\} \stackrel{d}{=}\left\{a^{H_{0}} X(t)+c(t)\right\}
$$

for some $a \in(0,1) \cup(1, \infty)$ and a nonrandom function $c:[0, \infty) \rightarrow \mathbb{R}^{d}$, then $\{X(t)\}$ is said to be wide-sense semi-selfsimilar.

Remark 2.2. The notion of (wide-sense) semi-selfsimilarity extends that of self-similarity (equality (1) is recovered if equality (2) holds for any $a>0$ and $c(t)=0$ ). As proved in [28], self-similar processes can be viewed as limiting processes of sequences of normalized processes, whereas semi-self-similar processes can be characterized as limiting processes of some sub-sequences of normalized processes. Wide sense semi-selfsimilarity is of course more flexible and can describe those circumstances where, for example, a first order stochastic dominance between distributions should be considered.

Theorem 2.1. (Taqqu, 1981) Let $\{X(t)\}$ be nontrivial, with stationary increments and selfsimilar with exponent $H_{0}$ (in notation $H_{0}$-sssi) and let $\mathbb{E}\left[|X(1)|^{2}\right]<\infty$. Then $\mathbb{E}[X(t) X(s)]=$ $\frac{1}{2}\left\{t^{2 H_{0}}+s^{2 H_{0}}-|t-s|^{2 H_{0}}\right\} \mathbb{E}\left[|X(1)|^{2}\right]$.

Remark 2.3. Theorem 2.1 provides a theoretical rationale for the rule of thumb used in financial practice to annualize volatility or to scale Value at Risk. In fact, from the theorem it readily follows $\mathbb{E}\left[X(t)^{2}\right]=t^{2 H_{0}} \mathbb{E}\left[|X(1)|^{2}\right]$. Assuming $X(t)$ to be the log-price of a stock, being $\mathbb{E}[X(t)] \simeq 0$, one as $\sigma^{2}(t) \simeq t^{2 H_{0}} \sigma^{2}(1)$, or - in terms of volatility $-\sigma(t) \simeq t^{H_{0}} \sigma(1)$. When Brownian motion is assumed to model the dynamic of log-price variation, one finally concludes $\sigma(t) \simeq t^{\frac{1}{2}} \sigma(1)$. More in general, for a $H_{0}$-sssi process, theorem 2.1 imply equality $\mathbb{E}\left[X(t)^{q}\right]=t^{H_{0} q} \mathbb{E}\left[|X(1)|^{q}\right]$.

- trivial, if $X(t)=X(0)$ almost surely for every $t>0$. 
For a process $X(t)$ with stationary increments the $a$-lagged increment sequence $Y(t, a):=$ $X(t+a)-X(t)$ satisfies $\{Y(t, a)\} \stackrel{d}{=}\{Y(s, a)\}$ for any $t$ and $s$. Therefore, if $X(t)$ is also $H_{0}$-sssi, with $H_{0}>0$, it follows that

$$
\begin{aligned}
\{X(a t)\} \stackrel{d}{=}\left\{a^{H_{0}} X(t)\right\} & \Rightarrow\{X(a)\} \stackrel{d}{=}\left\{a^{H_{0}} X(1)\right\} \Rightarrow \\
\{Y(0, a)\} \stackrel{d}{=}\left\{a^{H_{0}} Y(0,1)\right\} & \Rightarrow \quad\{Y(t, a)\} \stackrel{d}{=}\left\{a^{H_{0}} Y(t, 1)\right\} .
\end{aligned}
$$

Remark 2.4. Assuming that $X(t)$ is the log-price of a stock, i.e. $X(t)=\ln S(t)$ where $S(t)$ is the price at time $t$, the last equality of (3) states that the a-period log-return $Y(t, a):=\ln \frac{S(t)}{S(t-a)}$ distributes as the one-period log-return $Y(t, 1)=\ln \frac{S(t)}{S(t-1)}$, once this is scaled by $a^{H_{0}}$.

Our aim is to test the last equality of the chain (3).

In this concern, let us denote by $\mathcal{A}$ the compact set of time horizons, and let $\mathfrak{a}:=\min (\mathcal{A})<$ $\mathfrak{A}:=\max (\mathcal{A})$. For any $a \in \mathcal{A}$, let $\Phi_{Y(t, a)}(x)$ be the distribution of $\{Y(t, a)\}^{3}$. With this notation, equation (3) turns out to be

$$
\left.\Phi_{Y(t, a)}(x):=P(Y(t, a)<x)\right)=P\left(a^{H_{0}} Y(t, 1)<x\right)=\Phi_{a^{H_{0}} Y(t, 1)}(x),
$$

or, introducing for convenience the variable $H$, to

$$
\begin{aligned}
\Phi_{a^{-H} Y(t, a)}(x) & \left.:=P\left(a^{-H} Y(t, a)<x\right)\right) \\
& =P\left(a^{H_{0}-H} Y(t, 1)<x\right)=\Phi_{a^{H_{0}-H} Y(t, 1)}(x) \\
& =P\left(Y(t, 1)<a^{H-H_{0}} x\right)=\Phi_{Y(t, 1)}\left(a^{H-H_{0}} x\right)
\end{aligned}
$$

Let us denote now by $\Psi_{H}:=\left\{\Phi_{a^{-H} Y(t, a)}(x), a \in \mathcal{A}\right\}$ the set of the (absolutely continuous) distribution functions of $\left\{a^{-H} Y(t, a)\right\}$ and consider the distance function $\rho$ induced by the sup-norm $\|\cdot\|_{\infty}$ with respect to $\mathcal{A}$. The diameter of the metric space $\left(\Psi_{H}, \rho\right)$ is then defined as

$$
\delta\left(\Psi_{H}\right):=\sup _{x \in \mathbb{R}} \sup _{a_{i}, a_{j} \in \mathcal{A}}\left|\Phi_{a_{i}^{-H} Y\left(t, a_{i}\right)}(x)-\Phi_{a_{j}^{-H} Y\left(t, a_{j}\right)}(x)\right| .
$$

The notation introduced above allows to deduce the following results, proved in [5]:

Proposition 2.1. The process $Y$ is $H_{0}$-ss if and only if, for any $\mathcal{A} \subset \mathbb{R}^{+}, \delta\left(\Psi_{H_{0}}\right)=0$.

Proposition 2.2. Let $Y$ be $H_{0}$-ss. Then $\delta\left(\Psi_{H}\right)$ as a function of $H$ is non-increasing for $H \leq H_{0}$ and non-decreasing for $H \geq H_{0}$.

Being a necessary condition, Proposition 2.2: (a) implies that self-similarity can be rejected if the monotonicity with respect to $H$ does not occur; (b) combined with Proposition 2.1, it provides the self-similarity parameter as $H_{0}=\operatorname{argmin}_{H} \delta\left(\Psi_{H}\right)$.

\footnotetext{
${ }^{3}$ As usual, we will denote the density by $\phi$ and the distribution by $\Phi$. The results that will be recalled hold for the $d$-dimensional distributions of $X(t)$ and $Y(t, a)$ [5] [8], but the discussion will be circumscribed to the case $d=1$ in order to ease the connection with the empirical application of Section 5.
} 
Proposition 2.3. Let $Y$ be $H_{0}$-ss and let $\left\{\mathcal{A}_{n}\right\}$ be a sequence of compact sets such that $\mathfrak{a}_{i} \leq \mathfrak{a}_{j}$ and $\mathfrak{A}_{i} \geq \mathfrak{A}_{j}$ for $i>j$. Then, with respect to the sequence $\left\{\mathcal{A}_{n}\right\}, \delta\left(\Psi_{H}\right)$ is: (i) non-decreasing, if $\mathrm{H} \neq \mathrm{H}_{0}$; (ii) identically zero, if $\mathrm{H}=\mathrm{H}_{0}$.

By Proposition 2.3,

$$
\sup _{a_{i}, a_{j} \in \mathcal{A}}\left|\Phi_{a_{i}^{-H} Y\left(t, a_{i}\right)}(x)-\Phi_{a_{j}^{-H} Y\left(t, a_{j}\right)}(x)\right|=\left|\Phi_{\mathfrak{a}^{-H} Y(t, \mathfrak{a})}(x)-\Phi_{\mathfrak{A}^{-H} Y(t, \mathfrak{A})}(x)\right|
$$

and therefore the diameter (6) can be written as

$$
\begin{aligned}
\delta\left(\Psi_{H}\right) & :=\sup _{x \in \mathbb{R}}\left|\Phi_{\mathfrak{a}^{-H} Y(t, \mathfrak{a})}(x)-\Phi_{\mathfrak{A}^{-H} Y(t, \mathfrak{A})}(x)\right| \\
& =\sup _{x \in \mathbb{R}}\left|\Phi_{Y(t, 1)}\left(\mathfrak{a}^{H-H_{0}} x\right)-\Phi_{Y(t, 1)}\left(\mathfrak{A}^{H-H_{0}} x\right)\right| .
\end{aligned}
$$

provided that $Y$ is $H_{0}$-self-similar.

Since the last line of equation (3) can be generalized to any pair $a, b \in \mathcal{A}$, with $a \neq b$, as $\{Y(t, a)\} \stackrel{d}{=}\left\{\left(\frac{a}{b}\right)^{H_{0}} Y(t, b)\right\}$, using the notation introduced above, it is

$$
\begin{aligned}
\Phi_{Y(t, a)}(x) & =P(Y(t, a)<x) \\
& =P\left(\left(\frac{a}{b}\right)^{H_{0}} Y(t, b)<x\right)=\Phi_{\left(\frac{a}{b}\right)^{H_{0}} Y(t, b)}(x) \\
& =P\left(Y(t, b)<\left(\frac{b}{a}\right)^{H_{0}} x\right)=\Phi_{Y(t, b)}\left(\left(\frac{b}{a}\right)^{H_{0}} x\right)
\end{aligned}
$$

Thus, provided that $Y$ is semi-selfsimilar (or wide-sense selfsimilar), the scaling exponent is likely to change with $a, b$ and $t$. Therefore, given the set of time scales $\mathcal{A}=\{1,2, \ldots, K\}$, the number of pairs $(a, b), a \neq b$, with respect to which the self-similarity parameter $H(a, b, t)$ can be evaluated at any time $t$ is $\frac{K(K-1)}{2}$, since $H(a, b, t)=H(b, a, t)$. As a consequence, for any $t$ it is possible to build a (symmetric) matrix $\mathbb{H}(t)$ whose elements are $\mathbb{H}_{a, b}(t)=H(a, b, t)$. With the sole purpose to well-define $\mathbb{H}(t)$, we set $\mathbb{H}_{a, a}(t)=0$ since the values on the diagonal are not defined by virtue of (7). Because of its symmetry, $\mathbb{H}(t)$ can be redefined in terms of the associated strictly upper-triangular matrix, whose elements are

$$
\mathbb{H}_{a, b}(t)= \begin{cases}0 & \text { if } a \geq b \\ H(a, b, t) & \text { if } a<b\end{cases}
$$

Clearly, if $Y$ is a genuine $H_{0}$-ss process, $\mathbb{H}_{a, b}(t)=H_{0}$ for any $a<b$ and $t \geq 0$.

Remark 2.5. Although Proposition 2.1 states that $\delta\left(\Psi_{H_{0}}\right)=0$, actually the empirical diameter $\hat{\delta}\left(\Psi_{H_{0}}\right)$, rather than zero, turns out to be a non-negative random variable even for a genuine $H_{0}$-ss process. Therefore the statistical significance of $\hat{\delta}\left(\Psi_{H_{0}}\right)$ has to be evaluated. The general purpose to test the equality between two distributions is addressed by several nonparametric statistics available in literature: the Wilcoxon-Mann-Whitney (that detects 
Table 1: Statistical power values of the two-sample KS Test

for large sample sizes (with $\gamma_{2}=3.75, n_{1}=n_{2}=100$ )

\begin{tabular}{rrrrrrrrr}
\hline & $\gamma_{1=1.75}$ & $\gamma_{1=1.50}$ & $\gamma_{1=1.25}$ & $\gamma_{1=1.00}$ & $\gamma_{1=0.75}$ & $\gamma_{1=0.50}$ & $\gamma_{1=0.25}$ & $\gamma_{1=0.00}$ \\
\hline$\sigma_{1}=2 \sigma_{2}$ & 1,000 & 0.990 & 0.887 & 0.784 & 0.714 & 0.662 & 0.633 & 0.626 \\
$\sigma_{1}=3 \sigma_{2}$ & 1,000 & 1.000 & 1.000 & 0.999 & 0.997 & 0.994 & 0.993 & 0.991 \\
$\sigma_{1}=4 \sigma_{2}$ & 1,000 & 1.000 & 1.000 & 1.000 & 1.000 & 1.000 & 1.000 & 1.000 \\
\hline
\end{tabular}

both small and large samples. Their results indicate that, given the coefficient of kurtosis $\left(\gamma_{2}\right)$, the statistical power of KS test increases with the sample sizes, the coefficient of skewness $\left(\gamma_{1}\right)$ and the standard deviation ratios. Table 1 reproduces the power of the two-sample KS test only for large samples (which is indeed the case of our application).

\subsection{The case of fractional Brownian motion}

Equation (8) can be easily checked for the fractional Brownian motion, the Gaussian self-similar process par excellence. Using the wavelet-based simulator of $\mathrm{fBm}$ available in MatLab and built on the method described in [1] and modified by [3], three cases of fBm were surrogated with different parameters $\left(H_{0}=0.3,0.5,0.7\right)$. For each pair of timescales $(a, b) \in$ $\{1, \ldots, 125\}, a<b$, Figure 1 displays the surfaces generated by the $\alpha$-self-similarity matrices for a fixed time $t$. Since $\mathrm{fBm}$ is a genuine $H_{0}$-sssi process, for any time $t, \mathbb{H}(t)=H_{0}$, hence the surfaces are stationary with respect to time. The value ${ }^{\alpha} \hat{H}_{0}$ was calculated according to Propositions (2.1) and (2.2) as $\operatorname{argmin}_{H} \hat{\delta}\left(\Psi_{H}\right)$, provided that the distance between rescaled distributions was not statistically significant once the KS test was performed with $\alpha=5 \%$. As expected for a $\mathrm{H}_{0}$-ss process, the surfaces are fairly flat around a value about equal to the self-similarity parameter. The deviations that can be observed when the minimum and the maximum time scales tend to be close are mainly ascribable to the joint effect of the ratio $\frac{b}{a}$ in the r.h.s. of equation (8), which is close to 1 whatever the value of $H_{0}$, and of the accuracy 
of the simulator, which works effectively to capture the long range dependence rather than the short range one.
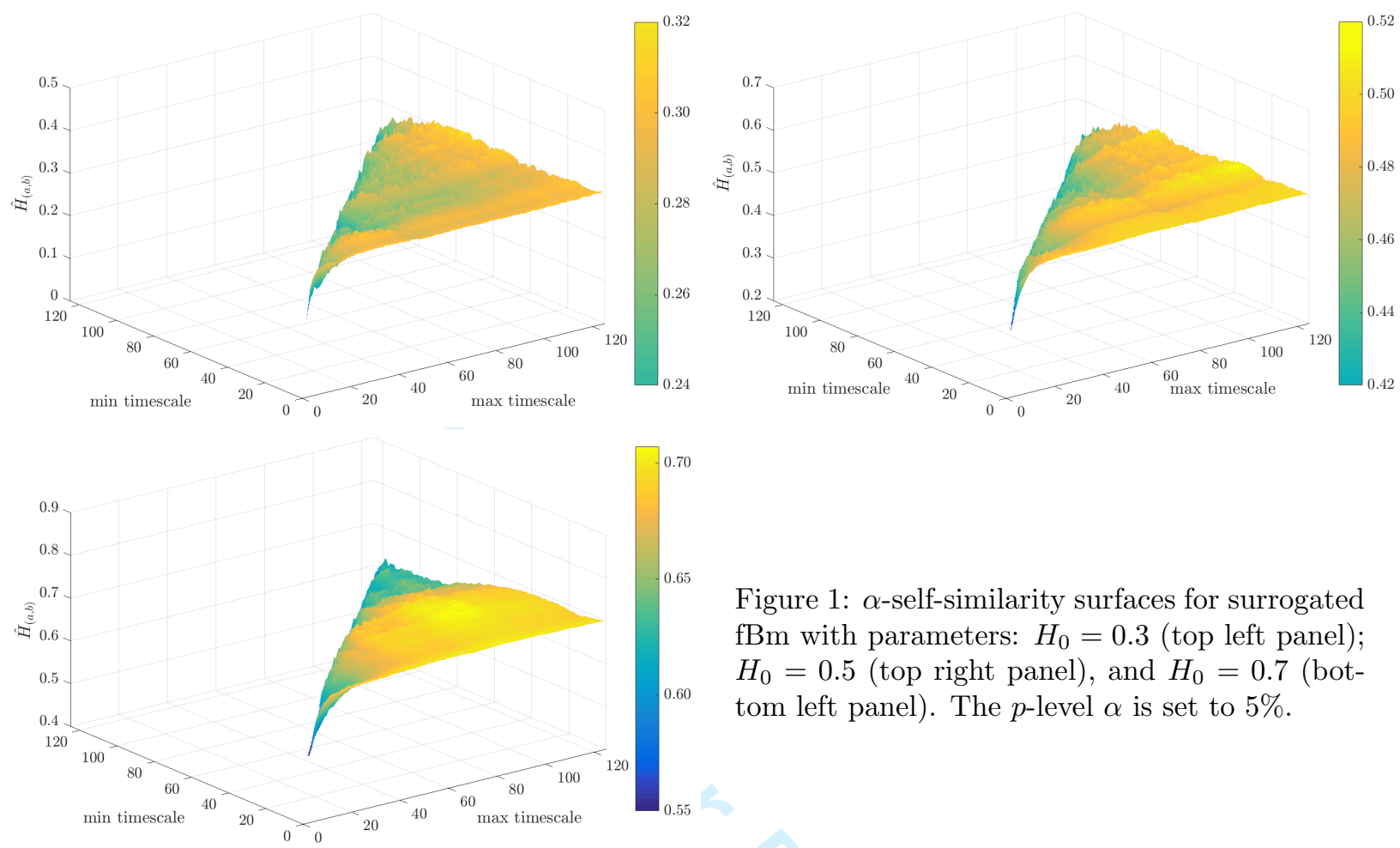

Figure 1: $\alpha$-self-similarity surfaces for surrogated fBm with parameters: $H_{0}=0.3$ (top left panel); $H_{0}=0.5$ (top right panel), and $H_{0}=0.7$ (bottom left panel). The $p$-level $\alpha$ is set to $5 \%$.

\section{Fractal Market Hypothesis, Liquidity and Distributions}

Unlike the EMH, the FMH emphasizes the role played by the heterogeneity of the investment horizons in providing liquidity to the market. In its original formulation, the FMH rests on the following five assumptions [33]:

(I) The market is made up of many individuals with a large number of different investment horizons.

(II) Information has a different impact on different investment horizons.

(III) The stability of the market is largely a matter of liquidity (balancing of supply and demand). Liquidity is available when the market is composed of many investors with many different investment horizons.

(IV) Prices reflect a combination of short-term technical trading and long-term fundamental valuation.

(V) If a security has no tie to the economic cycle, then there will be no long-term trend. Trading, liquidity, and short-term information will dominate.

Assumption (II) is the core of the FMH and it is also the quintessential of the notion of granularity described by [39]. The reasonable statement that the time scale dictates how market participants perceive and weigh the incoming information requires to be measured somehow. 
Since at an aggregate level it is not possible to know what kind of information each operator considers or what his individual reaction will be, the only possibility to measure the impact of the information is by evaluating its effects on the price variations. These are summarized by the whole distribution rather than by its specific moments. Therefore, despite it can appear more computationally demanding, the study of the self-similarity through the scaling of the entire distribution appears more correct and conceptually parsimonious, and justifies the approach developed in the previous Section.

In the light of the assumption that the link between self-similarity and liquidity is a matter of time resolution or granularity, the following example can illustrate the mechanism. For simplicity, let us assume the log-return of a stock to be normally distributed with mean zero and self-similar exponent $H=\frac{1}{2}$, and consider only two time scales: one day $(a=1)$ and twenty days $(b=20)$, corresponding to one trading month. To have simple calculations, let us assume the daily volatility to be $\sigma_{1}=1$; by self-similarity (see Remark 2.3), the monthly volatility is $20^{0.5} \cdot \sigma_{1}=20^{0.5} \simeq 4.47$. Now, let us assume that the daily trader experiences a large downward movement in the stock price, say an event of magnitude $-1.28 \sigma_{1}$ in terms of log-returns. Projecting this value on the daily distribution, the probability that the successive log-return will be negative equals $\Phi_{Y(t, 1)}(-1.28)=10 \%$. Since the complementary probability that the successive log-return will be positive equals $90 \%$, the daily trader may be willing to buy the stock, and his long position could easily match the short position of the monthly trader. Indeed, by self-similarity, the daily variation is equivalent on the monthly distribution to $38.72 \%$ of chances that the successive return will be negative. This follows from calculating $\Phi_{Y(t, 20)}(-1.28)$, with $Y(t, 20) \stackrel{d}{=} N\left(0,20^{0.5} \sigma_{1}\right)$ (see Figure 2). The effect is

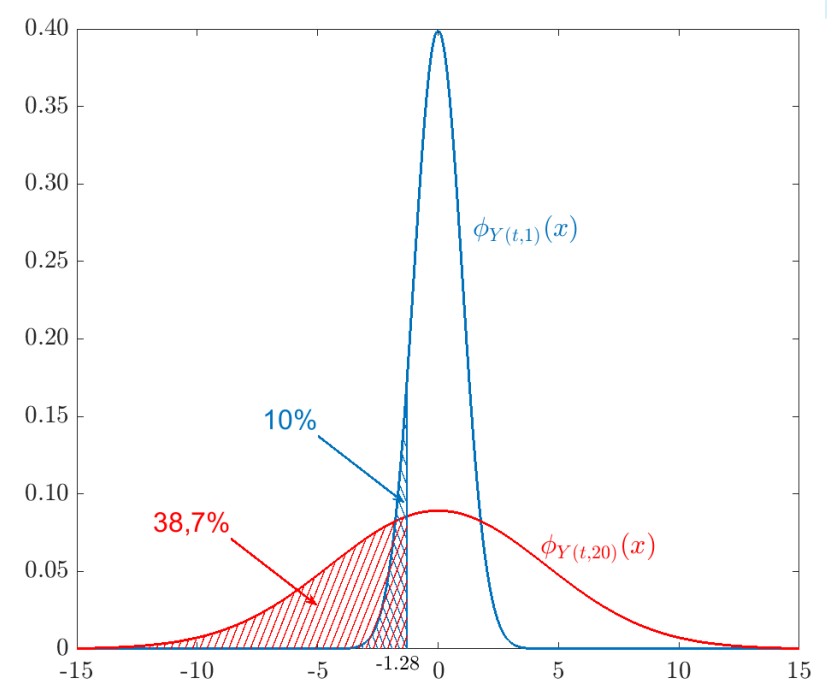

Figure 2: Densities $\phi_{Y(t, 1)}(x)$ and $\phi_{Y(t, 20)}(x)$ under the assumption of normality and $\frac{1}{2}$-ss. The liquidity originates by how the agents over the two different time scales evaluate the (conditional) probabilities of gain/loss with respect to the two different distributions. If self-similarity holds, no specific time scale characterizes or dominates the market, since scaling allows to compare relative probabilities among different investment horizons.

even more pronounced if the price process had a higher self-similarity parameter; for example, $\Phi_{Y(t, 20)}(-1.28)=43.75 \%$, if $Y(t, 20) \stackrel{d}{=} N\left(0,20^{0.7} \sigma_{1}\right)$. An opposite result occurs if the self-similarity parameter was low: $\Phi_{Y(t, 20)}(-1.28)=24.1 \%$, for $Y(t, 20) \stackrel{d}{=} N\left(0,20^{0.2} \sigma_{1}\right)$. Similar emphasized results could be observed if between the two scaled distributions a slight 
first order stochastic dominance occurred ${ }^{4}$. Here, the adjective slight is used to intend that the stochastic dominance should be sufficiently small to not reject self-similarity for a given $p$-value $\alpha$, but at the same time sufficiently large to increase the probability of conditional gain or loss of one investment horizon with respect to the other. Provided that the process is

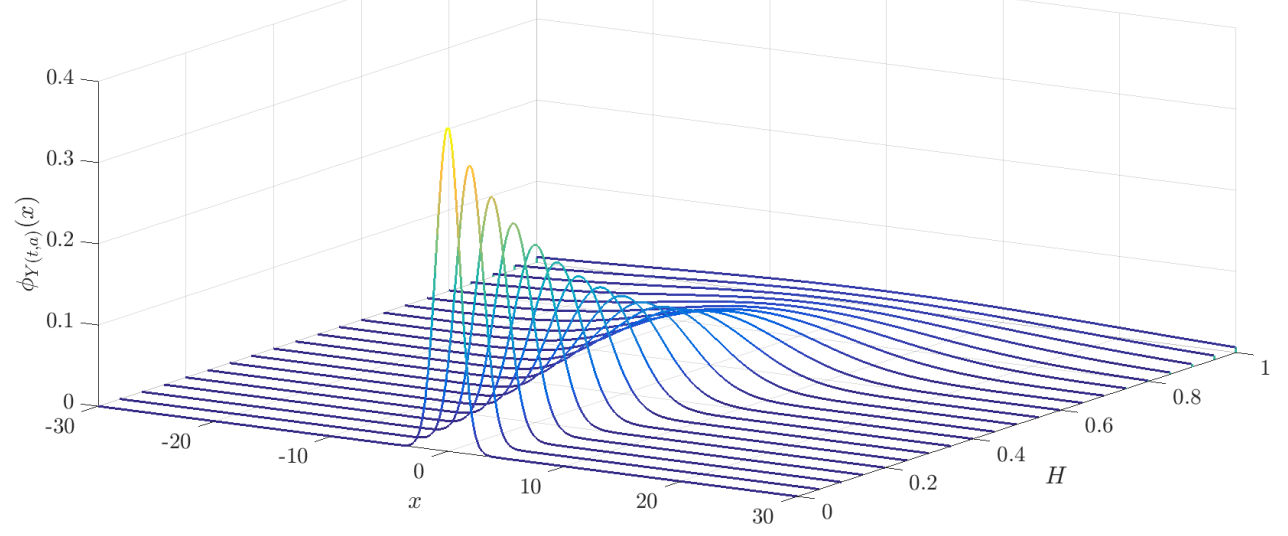

Figure 3: Normal densities $\phi_{Y(t, a)}(x)$ for different values of the scaling parameter $H$.

self-similar, Figure 3 shows how densities become fatter and fatter as the scaling parameter increases and suggests that the higher the value $H(a, b)$, the more liquid the fraction of the market operating at time scales $a$ and $b$. It is worthwhile to underline that the positive correlation between $H(a, b)$ and the liquidity is strongly consistent with the behavior of $H$ in terms of pointwise regularity of the process paths. Indeed, it is well known that the parameter of a self-similar stochastic process is inherently tied to the pointwise regularity of its paths, and that in some cases (e.g., the fBm) the two coincide; more in general, the higher the former, the higher the latter (see e.g., [7], [10], [17], [18], [34]). Smooth paths generally describe the case of a bull market, where the price is pushed by the existing liquidity that demands to be allocated; on the contrary, jagged paths depict the case of a (usually sudden) shock, triggered by a lack of liquidity which induces large downward variations in the market prices [6]. This phase is typically followed by a quick buy-and-sell trading activity that lasts until the impact of the new information has been metabolized by the market.

\section{Methodology}

\subsection{Directions to investors and regulators}

Given the mechanism described above, one relevant variable that can support the decision of market operators about the investment horizon to be preferred is the self-similarity parameter possibly linking each pair of time scales $a$ and $b$, for any time $t$. In fact, this can provide a signal of how much liquidity is connected to the chosen pair, because the higher the value of $H(a, b, t)$ the more potentially liquid the investment horizon, in absence of stochastic

\footnotetext{
${ }^{4}$ Recall that, given two cumulative distribution functions $F(x)$ and $G(x)$, defined for $x \in X$, $F$ first-order stochastically dominates $G$ if and only if $F(x) \leq G(x), \forall x \in X$.
} 
dominance effects. The mechanism is much more complex when one distribution stochastically dominates the other to such an extent that the self-similarity is rejected owing to the reciprocal location of the two distributions. This case has deep theoretical implications that deserve a separate discussion and therefore they will not be examined here, also because we do not expect statistically significant shifts in the rescaled distributions. The reason is that the data that will be considered in the application come from a unique stock index and not by different portfolios, for which a stochastic dominance analysis would be justified [12].

Besides the individual investors, the methodology we propose can provide public policymakers with tools that can contribute to stabilize markets during financial stress periods. In this regard, following [2], at least three main directions can be given:

a) Market-making guidelines, whose implementation is fraught with difficulty, also because of the undetermined size of the quotes at which the dedicated market makers should be required to transact [4] and about the capital requirements needed for the marketmakers activity. Transposed in the self-similarity matrix, the size of the quotes could be determined by looking at those investment horizons for which the estimated selfsimilarity index is very low.

b) Market "circuit breakers", that is the rules under which all trading of a given asset on a given exchange is halted if prices move too erratically with respect to some pre-set criteria. This was, for example, one of the issues analyzed by the Brady Commission Report following the crash of 1987, that we will discuss in Section 5. It is clear that according to our procedure a possible circuit breaker could be the intensity of diagonalization of the self-similarity matrix.

c) Minimum resting periods. They impose a minimum delay between the time at which a trade is submitted and that at which it is executed, with the effect of slowing trading down and preserving the confidence of longer-term investors. It is a decision involving a trade-off, since - increasing the transaction costs by high-frequency traders - minimum resting periods may have an adverse effect on liquidity, at least during normal times. Therefore, it is clear that delays could be imposed when the self-similarity matrix reveals holes (i.e. the KS test is rejected) or very low values of $H(a, b)$ for large $a$ and $b$ as compared with high values of $H(a, b)$ for small $a$ and $b$.

\subsection{Implementation}

In order to estimate the self-similarity parameter, it should be observed that equations (7) and (8) refer to the stochastic process, but actually only one trajectory $X(t)=\ln S(t)$, $t=1, \ldots, T$, can be observed. Therefore, only one value would correspond to any fixed time $t$ and no distribution could be calculated. To circumvent this inherent limit of the data, for any $t$, let us denote by $\mathbf{t}_{\Delta}^{s}:=(t+s, t+s+1, \ldots, t+\Delta)$ the time window of length $\Delta-s+1$ starting from time $t$; within this, we calculated the log-price variations from lag 1 to lag $\Delta / 2$, that is $Y\left(\mathbf{t}_{\Delta}^{1}, 1\right), Y\left(\mathbf{t}_{\Delta}^{2}, 2\right), \ldots, Y\left(\mathbf{t}_{\Delta}^{\Delta / 2}, \Delta / 2\right)$. The choice to set the maximum lag $\mathfrak{A}=\Delta / 2$ was motivated by the need to have a sufficient number of data to shape the empirical distribution $\hat{\Phi}_{Y\left(\mathbf{t}_{\Delta}^{\mathfrak{A}}, \mathfrak{A}\right)}(x)$. For any pair $(a, b)$, with $a=1, \ldots, \mathfrak{A}-1$ and $b=a+1, \ldots, \mathfrak{A},{ }^{\alpha} H\left(a, b, \mathbf{t}_{\Delta}^{0}\right)$ was calculated as

$$
{ }^{\alpha} \hat{H}\left(a, b, \mathbf{t}_{\Delta}^{0}\right)=\arg \min _{H \in[0,1)} \hat{\delta}\left(\Psi_{H}\right)
$$


where

$$
\hat{\delta}\left(\Psi_{H}\right)=\max _{x \in \mathbb{R}}\left|\hat{\Phi}_{Y\left(\mathbf{t}_{\Delta}^{a}, a\right)}(x)-\hat{\Phi}_{Y\left(\mathbf{t}_{\Delta}^{b}, b\right)}\left((b / a)^{H} x\right)\right| .
$$

The $p$-value $\alpha$ refers to the nonparametric two-sample Kolmogorov-Smirnov statistic, which tests the null hypothesis that $\hat{\Phi}_{Y\left(\mathbf{t}_{\Delta}^{a}, a\right)}(x)$ and $\hat{\Phi}_{Y\left(\mathbf{t}_{\Delta}^{b}, b\right)}\left((b / a)^{H_{0}} x\right)$ are equal. The use of this test appears as natural choice since, like the KS statistic, the diameter is defined as the maximum absolute difference between the empirical cumulative distribution functions (see Remark 2.5). Thus, for $t=1, \ldots, T-\Delta$, we finally obtain the sequence of estimated $\alpha$-self-similarity matrices ${ }^{\alpha} \hat{\mathbb{H}}\left(\mathbf{t}_{\Delta}^{0}\right)$ whose elements ${ }^{\alpha} \hat{\mathbb{H}}_{a, b}\left(\mathbf{t}_{\Delta}^{0}\right)$ are ${ }^{\alpha} \hat{H}\left(a, b, \mathbf{t}_{\Delta}^{0}\right)$ with the change of $a$ and $b$.

As discussed in Section 3, the larger the value ${ }^{\alpha} \hat{\mathbb{H}}_{a, b}\left(\mathbf{t}_{\Delta}^{0}\right)$ the larger the contribution to the overall liquidity of the investment horizons identified by the pair $(a, b)$. Since each market operator decides only his own investment horizon whereas it is irrelevant to know on which horizon his counterpart in a trade is acting, the knowledge of the overall liquidity connected to each horizon can ease the selection of the more liquid investment horizon. In this regard, a natural and immediate measure of the liquidity potentially available for each horizon $a$ in the time window $\mathbf{t}_{\Delta}^{0}$ can be defined as

$$
{ }^{\alpha} \mathcal{L}_{a}\left(\mathbf{t}_{\Delta}^{0}\right)=\sum_{i=1}^{a}{ }^{\alpha} \hat{\mathbb{H}}_{i, a}\left(\mathbf{t}_{\Delta}^{0}\right)+\sum_{j=a+1}^{\mathfrak{A}}{ }^{\alpha} \hat{\mathbb{H}}_{a, j}\left(\mathbf{t}_{\Delta}^{0}\right), \quad a=1, \ldots, \mathfrak{A}-1
$$

For any investment horizon $a,{ }^{\alpha} \mathcal{L}_{a}\left(\mathbf{t}_{\Delta}^{0}\right)$ is nothing but the sum of the $a^{\text {th }}$ row and $a^{\text {th }}$ column of matrix ${ }^{\alpha} \hat{\mathbb{H}}\left(\mathbf{t}_{\Delta}^{0}\right)$.

\section{Application}

\subsection{Data}

The methodology described in the previous Section was implemented to analyze the U.S. daily stock index S\&P500 from January $3^{\text {rd }}, 1950$ to August $1^{\text {st }}, 2016$ (16, 753 observations). The adjusted close was considered as the basis to calculate the log-index variations. The length $\Delta$ of the time window was set to 250 , corresponding to about one trading year. In this way, calculating the log-price variations from $\mathfrak{a}=1$ up to $\mathfrak{A}=125$ trading days, a minimum of 125 data were available to shape the empirical cumulative distribution of the largest time scale. In order to preserve at the best the raw data and to avoid spurious effects, no fitting was used to regularize the empirical distributions served as input for the KS test, whose $p$-value was set at $\alpha=0.05$. The choice to consider a very long time series is obviously not a requirement of the methodology. Rather, it was motivated by the necessity to analyze the self-similarity matrices before, during and after the main financial events in order to establish the possible existence of patterns independent on local contingencies. Tables 2 and 3 list, respectively, the events corresponding to the daily variations above $5 \%$ and below $-5 \%$. The dynamical analysis was performed for the entire sample with a time step of five days (one trading week), so to obtain a sequence made of 3,301 self-similarity matrices of order 125 . For brevity, here only the four largest financial events will be extensively documented and discussed, with the understanding that the analysis produces similar results in the other cases that were examined as well. In particular, since the first two events (the 2007-2009 financial 
Table 2: S\&P500 (1950-2016): Positive variations larger than 5\%

Table 3: S\&P500 (1950-2016): Negative variations smaller than $-5 \%$

\begin{tabular}{rrlrrll}
\hline Rank & Year & Date & Close & Change & Weekday & Name \\
\hline 1. & 2008 & $13 / 10 / 2008$ & $1,003.35$ & 11.58 & Monday & 200\%-2009 financial crisis \\
2. & 1987 & $21 / 10 / 1987$ & 258.38 & 9.10 & Wednesday & Black Monday \\
3. & 2009 & $23 / 03 / 2009$ & 822.92 & 7.08 & Monday & American Recovery and Reinvestment Act \\
4. & 2002 & $24 / 07 / 2002$ & 843.43 & 5.73 & Wednesday & After-fall rally \\
5. & 1997 & $28 / 10 / 1997$ & 921.85 & 5.12 & Tuesday & Tom Yum Goong recover \\
6. & 1998 & $08 / 09 / 1998$ & $1,023.46$ & 5.09 & Tuesday & Russian financial crisis recover \\
7. & 1970 & $27 / 05 / 1970$ & 72.77 & 5.02 & Wednesday & Cambodian Campaign \\
8. & 2001 & $03 / 01 / 2001$ & $1,347.56$ & 5.01 & Wednesday & Dot-com bubble \\
\hline
\end{tabular}

crisis and the Black Monday) are common to both the Tables, we will examine these along with one further event for the positive variations (American Recovery and Reinvestment Act) and one further event for the negative variations (Tom Yum Goong mini-crash).

\begin{tabular}{|c|c|c|c|c|c|c|}
\hline Rank & Year & Date & Close & Change & Weekday & Name \\
\hline 1. & 1987 & $\begin{array}{l}19 / 10 / 1987 \\
\end{array}$ & 224.84 & -20.47 & Monday & Black Monday \\
\hline 2. & 2008 & $15 / 10 / 2008$ & 907.84 & -9.03 & Wednesday & 2007-2009 financial crisis \\
\hline 3. & 1997 & $27 / 10 / 1997$ & 876.99 & -6.87 & Monday & Tom Yum Goong mini-crash \\
\hline 4. & 1998 & $31 / 08 / 1998$ & 957.28 & -6.80 & Monday & Russian financial crisis \\
\hline 5. & 1988 & $08 / 01 / 1988$ & 243.40 & -6.77 & Friday & Brady Report on market mechanism \\
\hline 6. & 1962 & $28 / 05 / 1962$ & 55.50 & -6.68 & Monday & Kennedy Slide \\
\hline 7. & 2011 & $08 / 08 / 2011$ & $1,119.46$ & -6.68 & Monday & Credit Rating Crisis \\
\hline 8. & 1955 & $26 / 09 / 1955$ & 42.61 & -6.62 & Monday & President Eisenhower's heart attack \\
\hline 9 . & 1989 & $13 / 10 / 1989$ & 333.65 & -6.12 & Friday & Mini Black Friday \\
\hline 10. & 2000 & $14 / 04 / 2000$ & $1,356.56$ & -5.83 & Friday & Bleak Friday \\
\hline 11. & 1950 & $26 / 06 / 1950$ & 18.11 & -5.38 & Monday & Korean War \\
\hline 12. & 2009 & $20 / 01 / 2009$ & 805.22 & -5.28 & Tuesday & US bear market 2007-2009 \\
\hline
\end{tabular}

\subsection{Results}

Figures from 4 to 7 display the estimated self-similarity surfaces, before (top left panel), during (top right panel) and after (bottom left panel) the analyzed financial event. The bottom right panel summarizes the index S\&P500 in the time span considered to build the matrices. 
Figure 4 (Black Monday). On October 19, 1987, stock markets around the world crashed. Spread from Hong Kong to Europe and the United States, the crash caused a drop in the S\&P500 of 57.86 points (-20.47\%). A mixture of automatic trading, overvaluation, illiquidity and market psychology was indicated as the cause of the crash. Even if controversial, the role of automatic trading as the main cause of the shock is largely shared.

The analysis shows that before the event the market was characterized by moderately high values in the self-similarity parameter up to about 60 trading days and very low values in the horizon $60-125$ days. In correspondence of the flash crash, $77.4 \%$ of the pairs do not pass the KS test and the matrix becomes therefore almost diagonal, virtually zeroing the liquidity of the whole market. After the crash, the market slowly recovered its scaling structure, even if the values appear decidedly lower than before the event.
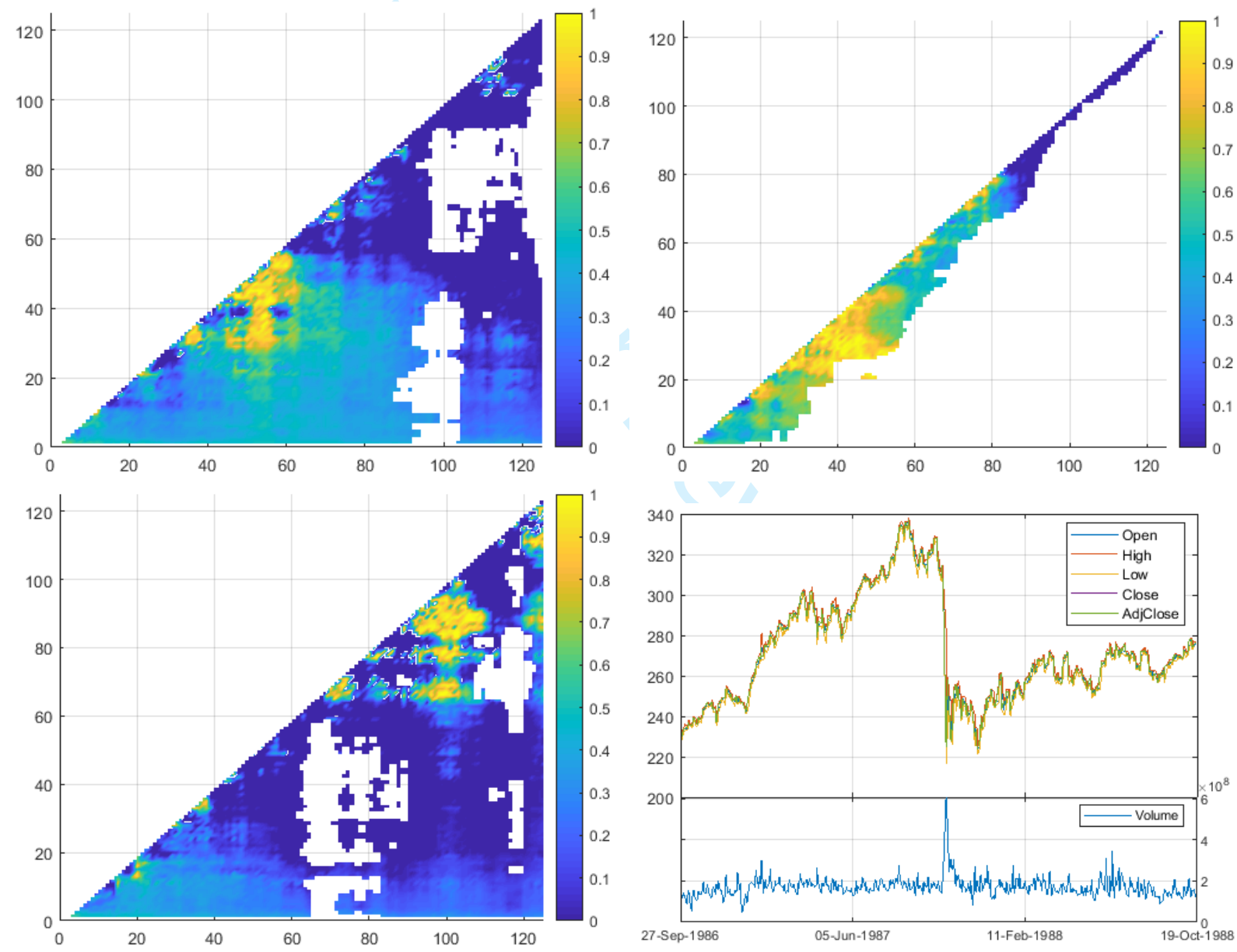

Figure 4: Top left panel: 0.05 -self-similarity surface before the event. Top right panel: 0.05 -self-similarity surface during the event. Bottom left panel: 0.05-self-similarity surface after the event. Bottom right panel: S\&P500 index. 
Figure 5 (2007 - 2009 financial crisis). On October 13, 2008 stock markets worldwide rose and the S\&P500 reached $1,003.35$ points $(+11.58 \%)$, as a consequence of the plan - announced by the U.S. government - to take an equity interest of $\$ 250$ billion in U.S. banks. The 9 largest banks, called into a meeting on Monday morning, were pressured to sign. The rationale of the plan was that the additional capitalization and the guarantees could help the banks to reactivate a normal lending pattern with each other and borrowers. On October 15 the S\&P500 again collapsed down over 9\%.

Before the event the market was characterized by very high values of $H(a, b)$ in the range 20 - 40 trading days, moderately high values between 40 and 70 days, and very low values in the horizon $70-125$ days. During the crisis the scaling structure heavily fractured; the KS test is rejected in $55.0 \%$ of the cases, and self-similarity continued to hold only for the shortest time scales. After the intense drop of October 2009, the market started recovering its scaling structure and, significantly, as denoted by the higher values of $H(a, b)$, the liquidity increased before on the longer time horizons and then it "propagated" to the shorter ones.
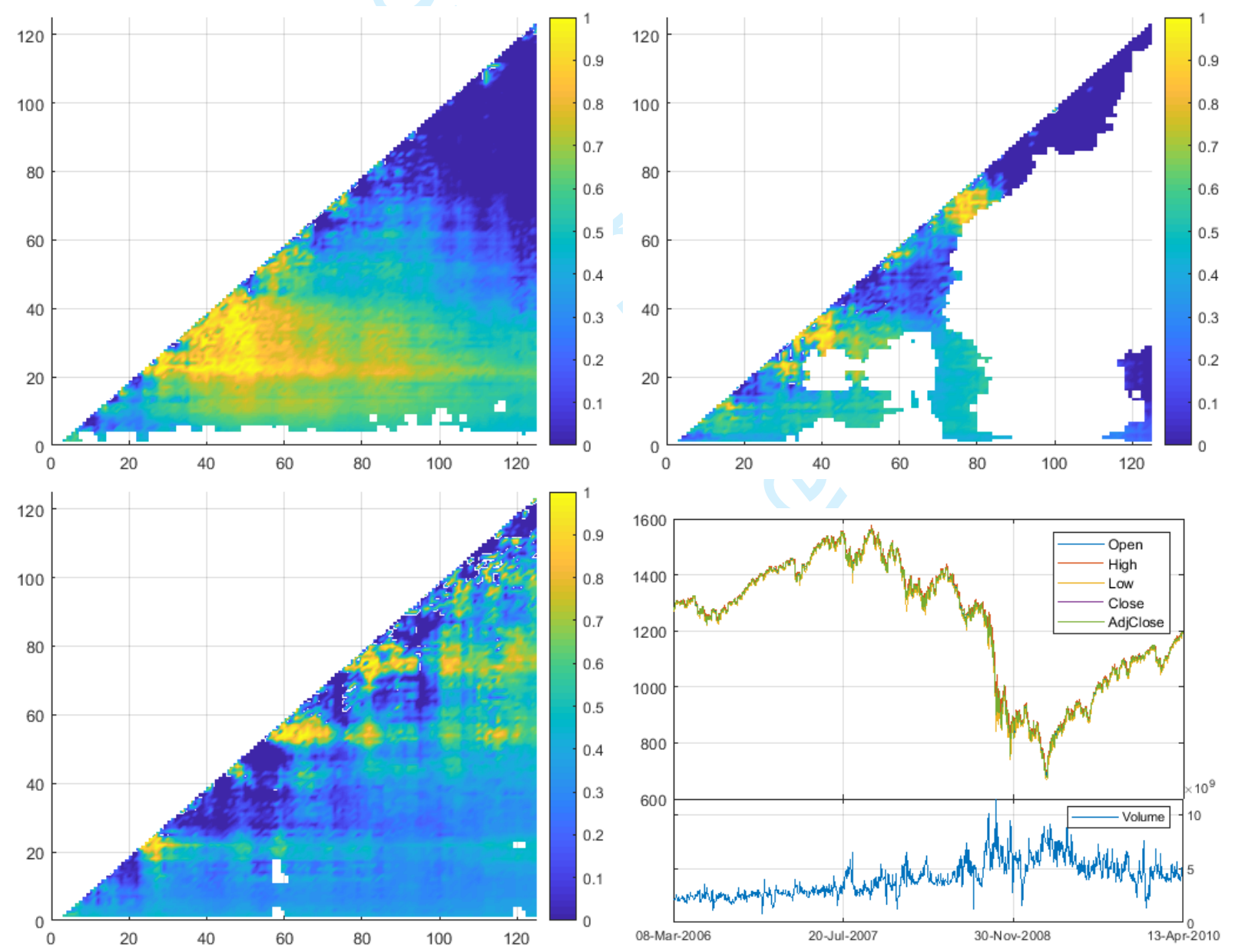

Figure 5: Top left panel: 0.05-self-similarity surface before the event. Top right panel: 0.05-self-similarity surface during the event. Bottom left panel: 0.05-self-similarity surface after the event. Bottom right panel: S\&P500 index. 
Figure 6 (American Recovery and Reinvestment Act). Stock markets surged after the news - released on March 23, 2009 - about the Treasury's plan to buy large amounts of junk bank assets and a better-than-expected existing home sales report. The plan was an economic stimulus package of $\$ 787$ billion. It provided for tax relief, expansion of unemployment benefits, social assistance provisions, national spending on education, health care, and infrastructure, including the energy sector. The S\&P 500 rose 54 points $(+7.8 \%)$, its best one-day gain since November 13, 2008. Since its tumbling to 12-year lows of the previous two weeks, the S\&P 500 rallied $18 \%$.

A pattern different from the previous two cases can be observed in this case. Before the launch of the ARRA, a large portion of the investment horizons (mainly the long-term ones) lacked its self-similarity structure, and the matrix appeared almost diagonal, with moderately high values of $H(a, b)$. Only $45.2 \%$ of the data passed the KS test. The situation decidedly improved with the launch of the stimulus package, whose effects lasted for several months, increasing the liquidity especially on the longer time horizons (notice the very high values of the self-similarity parameter in the range $60-80$ trading days).
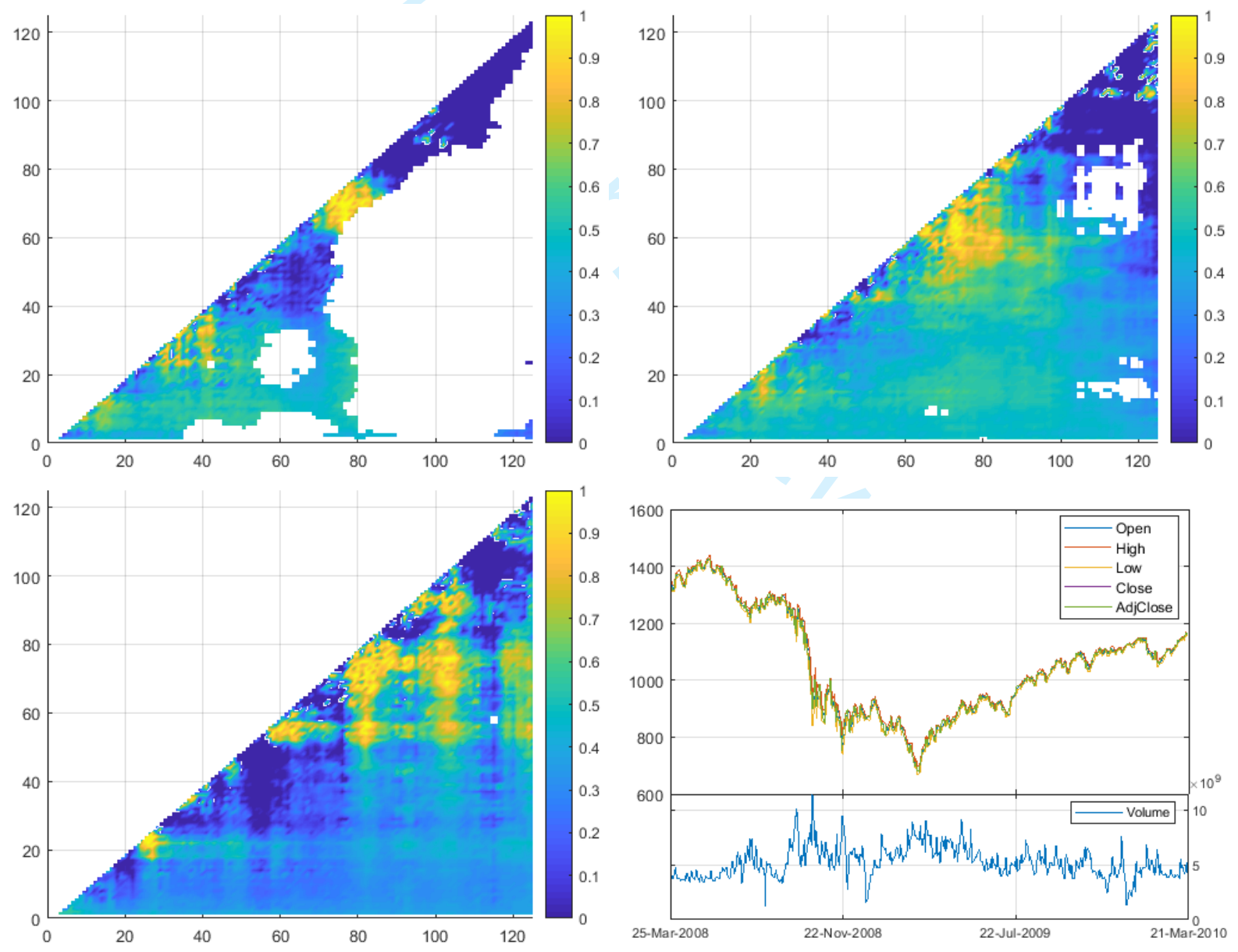

Figure 6: Top left panel: 0.05 -self-similarity surface before the event. Top right panel: 0.05 -self-similarity surface during the event. Bottom left panel: 0.05-self-similarity surface after the event. Bottom right panel: S\&P500 index. 
Figure 7 (Tom Yum Goong mini-crash). On October 27,1997 the collapse of the Thai baht, caused by the government decision to float it to support its currency peg to the U.S. dollar, triggered a chain reaction in the world markets. Most of Southeast Asia and Japan experienced slumps in their currencies, drops in stock markets, and a huge rise in private debt. The crisis started overnight with a fall of the Hang Seng Index of $6 \%$ and then spread to the European and U.S. markets. The S\&P500 closed at 876.99 points $(-6.87 \%)$.

Interestingly, the crisis is known as "mini-crash". Indeed, the three similarity matrices are quite similar, except for the fact that, after the mini-crash, higher estimates of $H(a, b)$ were reached on most of the time horizons. These go along with a lower rate of rejection of the KS test (9.5\%, compared to $20.1 \%$ before and $22.3 \%$ during the crash). The homogeneity among the three matrices is also strongly consistent with the historical reconstruction according to which, despite the crisis marked the end of the 1990s economic boom in the U.S. and Canada, both consumer confidence and economic growth were only marginally affected during the winter of $1997-98$, compared to the rest of the world. When both returned to pre-October levels, the index came back growing, even if at a slower pace than before the crash.
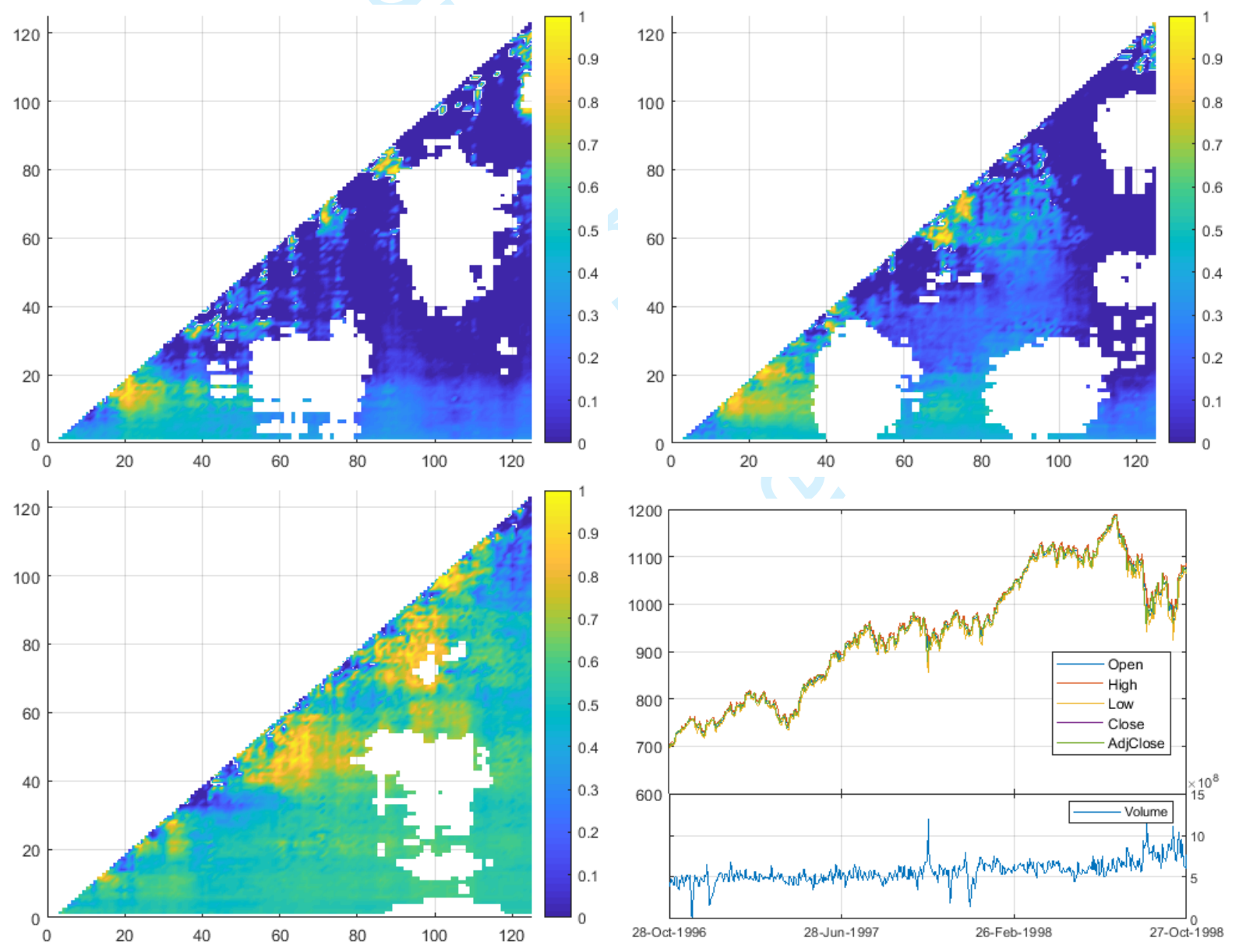

Figure 7: Top left panel: 0.05 -self-similarity surface before the event. Top right panel: 0.05 -self-similarity surface during the event. Bottom left panel: 0.05-self-similarity surface after the event. Bottom right panel: S\&P500 index. 

4-7. In all cases, the index captures the liquidity regime switching in the neighborhood of the considered financial event. For example, in the top left panel (Black Monday) it is evident that before the crash the liquidity was lacking up to 20 trading days (A), whereas it was higher from 20 to 60 days (B) and then again low (C). The situation is reversed immediately after the crash, when liquidity increases on the shorter investment horizons, diminishes on the medium term and increases a lot in the long ones. Also the top right and the bottom left panels reveal bursts of liquidity for all the investment horizons in correspondence with the U.S. government plan to restablish market liquidity (A) and the end of the Term Auction Facility program (B). In particular, top right panel highlights also the lack of liquidity on longer investment horizons during the 2007-2009 global financial crisis (C). Finally, bottom right panel shows that the Asian crisis, reflected in the market drop of October 27, 1997, was indeed a mini-crash, since neither the liquidity nor the scaling structure of the market were heavily affected by this event.
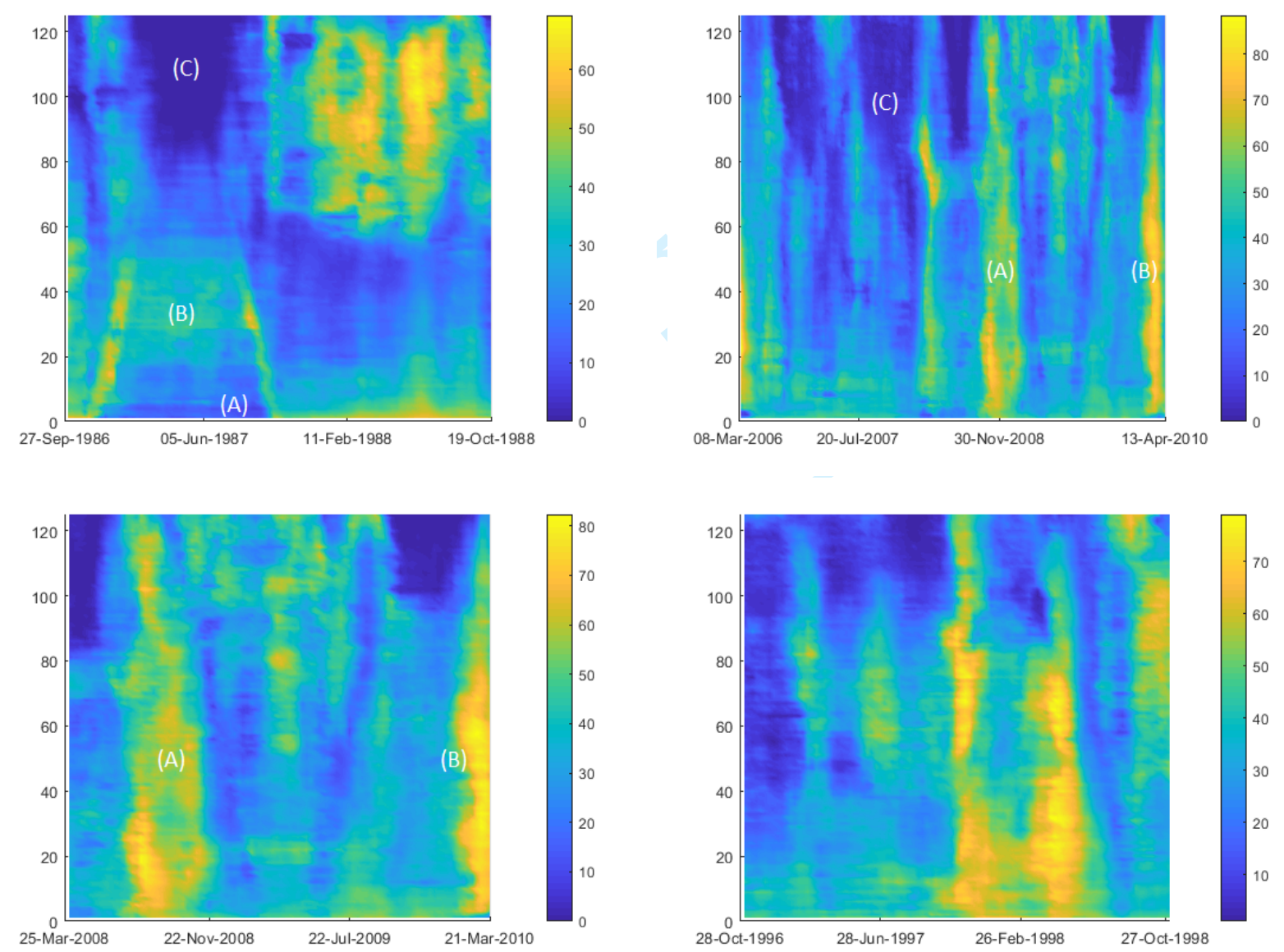

Figure 8: ${ }^{0.05} \mathcal{L}_{a}$ for $a=1, \ldots, 125$. Top left panel: Black Monday. Top right panel: $2007-2009$ financial crisis. Bottom left panel: American Recovery and Reinvestment Act. Bottom right panel: Tom Yum Goong crisis. 


\section{Conclusions}

In this work a new method is proposed to analyze scale invariance and to eventually estimate the self-similarity parameter that characterizes any pairs of investment horizons. The methodology enriches the existing ones because, instead of considering how specific moments scale with respect to the time frequency sampling, it considers the distributions as a whole. The method is nonparametric and rests on previous theoretical results which permit to estimate the exponent of a self-similar process by inverting the diameter of the space of rescaled distributions. Within the framework of the Fractal Market Hypothesis, given the relation between scale invariance, value of the self-similarity exponent and liquidity in the stock market, we can mark out the contribution of each investment horizon to the overall liquidity of the market. The results of an empirical application to the index S\&P500 show that our procedure effectively captures how the market scaling structure and liquidity evolve in correspondence with the main financial events, thus revealing a very complex, time-changing dynamics. The self-similarity matrices that we define classify the investment horizons in terms liquidity, and therefore they ultimately represent a way to measure the illiquidity risk. Even more relevant, they can contribute to design the guidelines that public policymakers could adopt to bolster market stability.

Our analysis can be deepened along at least two directions:

- it is well known that the KS test displays low statistical power with respect to other alternatives. This suggests to study more in detail the sensitiveness of the results to the power and, even more, to design a comparative analysis of several statistical tests. In this regard, a different approach to evaluate the statistical significance of diameter (7) is represented by the empirical likelihood ratio introduced by [19], which should reduce the standard deviation of the estimates.

- the model's power to forecast of conditional liquidity constitutes a second relevant strand. In this regard, the challenge is to extract from data conditional distributions not affected by the inertia induced by the moving-window effect. This could be achieved by using multidimensional data instead of the (one-dimensional) market index. In fact, the use of individual stocks would allow to have at any time $t$ a dataset sufficient to shape the empirical distribution, bypassing in this way the use of a time window.

\section{Acknowledgement}

The Authors thank the anonymous Reviewers for their helpful comments and suggestions.

\section{References}

[1] Abry, P., Sellan, F., 1996. The wavelet-based synthesis for the fractional brownian motion proposed by f. sellan and y. meyer: Remarks and fast implementation. Appl. Comp. Harmonic Anal. 3 (4), 377-383.

[2] Anderson, N., Noss, J., 2013. The fractal market hypothesis and its implications for the stability of financial markets. Tech. rep., Bank of England, Financial Stability Paper n.23. 
[3] Bardet, J.-M., Lang, G., Oppenheim, G., Philippe, A., Stoev, S., Taqqu, M., 2003. Generators of long-range dependence processes: a survey. Birkhäuser, pp. 579-623.

[4] Benos, E., Wethrilt, A., 2012. The role of designated marketmakers in the new trading landscape. Bank of England Quarterly Bulletin 52 (4), 343-353.

[5] Bianchi, S., 2004. A new distribution-based test of self-similarity. Fractals 12 (03), 331346.

[6] Bianchi, S., Frezza, M., 2018. Liquidity, efficiency and the $2007-2008$ global financial crisis. Annals of Economics and Finance 19 (2), 375-404.

[7] Bianchi, S., Pantanella, A., Pianese, A., 2013. Modeling stock prices by multifractional brownian motion: an improved estimation of the pointwise regularity. Quantitative Finance 13 (8), 1317-1330.

[8] Bianchi, S., Pantanella, A., Pianese, A., Palazzo, A., 2013. Self-similarity parameter estimation for k-dimensional processes. International Journal of Computer Theory and Engineering 5, 302-306.

[9] Bianchi, S., Pianese, A., 2008. Multifractional properties of stock indices decomposed by filtering their pointwise hlder regularity. Int. J. Theor. Appl. Fin. 11 (6), 567-595.

[10] Bianchi, S., Pianese, A., 2014. Multifractional processes in finance. Risk and Decision Analysis 5 (1), 1-22.

[11] Bouchaud, J.-P., Potters, M., 2000. Theory of Financial Risk and Derivative Pricing. From Statistical Physics to Risk Management. Cambridge University Press.

[12] Clark, E., Kassimatis, K., 2014. Exploiting stochastic dominance to generate abnormal stock returns. Journal of Financial Markets 20, 20 - 38.

[13] Cont, R., Potters, M., Bouchaud, J.-P., 1997. Scaling in stock market data: Stable laws and beyond. In: Dubrulle, B., Graner, F., Sornette, D. (Eds.), Scale Invariance and Beyond. Springer Berlin Heidelberg, Berlin, Heidelberg, pp. 75-85.

[14] Dar, A., Bhanja, N., Tiwari, A., 2017. Do global financial crises validate assertions of fractal market hypothesis? International Economics and Economic Policy 14 (1), 153165.

[15] Doorasamy, M., Sarpong, K., 2018. Fractal market hypothesis and markov regime switching model: A possible synthesis and integration. International Journal of Economics and Financial Issues 8 (1), $93-100$.

[16] Fama, E., 1965. The behavior of stock-market prices. The Journal of Business 38 (1), $34-105$.

[17] Frezza, M., 2012. Modeling the time-changing dependence in stock markets. Chaos, Solitons \& Fractals 45, 1510-1520. 
[18] Frezza, M., 2018. A fractal-based approach for modeling stock price variations. Chaos 28 (091102), 1-6.

[19] Gurevich, G., Vexler, A., Oct 2011. A two-sample empirical likelihood ratio test based on samples entropy. Statistics and Computing 21 (4), 657-670.

[20] Hasbrouck, J., Schwartz, R., 1988. Liquidity and execution costs in equity markets. The Journal of Portfolio Management 14 (3), 10-16.

[21] Karp, A., Van Vuuren, G., 2019. Investment implications of the fractal market hypothesis. Annals of Financial Economics 14 (01), 1950001.

[22] Kolmogorov, A., 1933. Sulla determinazione empirica di una legge di distribuzione. Giornale dellistituto Italiano degli Attuari 4, 83-91.

[23] Kolmogorov, A., 1941. Confidence limits for an unknown distribution function. Annals of Mathematical Statistics 12, 461-463.

[24] Kristoufek, L., 2012. Fractal markets hypothesis and the global financial crisis: scaling, investment horizons and liquidity. Advances in Complex Systems 15 (6), 1250065.

[25] Kristoufek, L., 2013. Fractal markets hypothesis and the global financial crisis: wavelet power evidence. Scientific Reports 3, 2857.

[26] Kumar, A., Jayakumar, C., Kamaiah, B., 2017. Fractal market hypothesis: evidence for nine asian forex markets. Indian Economic Review 52, 181-192.

[27] Landoni, E., Ambrogi, F., Mariani, L., Miceli, R., 2016. Parametric and nonparametric two-sample tests for feature screening in class comparison: a simulation study. Epidemiology Biostatistics and Publich Health 13 (2), e11808-1/11.

[28] Maejima, M., Sato, K., 1999. Semi-selfsimilar processes. Journal of Theoretical Probability $12(2), 347-373$.

[29] Mandelbrot, B., 1963. The variation of certain speculative prices. Journal of Business XXXVI, 392-417.

[30] Mandelbrot, B., 1997. Fractals and Scaling in Finance. Discontinuity, Concentration, Risk Selecta Volume E. Springer.

[31] Mandelbrot, B., Taylor, H., 1967. On the distribution of stock price differences. Operations Research 15, 1057-1062.

[32] Mantegna, R., Stanley, H., 2004. An Introduction to Econophysics. Correlations and Complexity in Finance. Cambridge University Press.

[33] Peters, E., 1994. Fractal market analysis Applying chaos theory to investment and analysis. Wiley. 
[34] Pianese, A., Bianchi, S., Palazzo, A., 2018. Fast and unbiased estimator of the timedependent hurst exponent. Chaos 28 (31102), 1-6.

[35] Rachev, Z., Weron, A., Weron, R., 1999. Ced model for asset returns and fractal market hypothesis. Math Comput Model 29 (1), 23-36.

[36] Samuelson, P., 1965. Rational theory of warrant pricing. Industrial Management Review $6(2), 13-39$.

[37] Senger, Ö., Çelik, A., 2013. A monte carlo simulation study for kolmogorov-smirnov two-sample test under the precondition of heterogeneity: upon the changes on the probabilities of statistical power and type i error rates with respect to skewness measure. Journal of Statistical and Econometric Methods 2 (4), 1-15.

[38] Smirnov, N., 1948. Table for estimating the goodness of fit of empirical distributions. Annals of Mathematical Statistics 19 (2), 279-281.

[39] Tapiero, C. S., Tapiero, O. J., Jumarie, G., 2016. The price of granularity and fractional finance. Risk and Decision Analysis 6 (1), 7-21.

[40] Weron, A., Weron, R., 2000. Fractal market hypothesis and two power-laws. Chaos Solitons \& Fractals 11 (1), 289 - 296. 